\title{
CARACTERIZACIÓN MECÁNICA Y MORFOLÓGICA DE TERMOPLÁSTICOS RECICLADOS ESPUMADOS REFORZADOS CON SUB PRODUCTOS DE MADERA
}

\section{MORPHOLOGICAL AND MECHANICAL CARACTERIZATION OF RECYCLED THERMOPLASTIC FOAMS REINFORCED WITH WOOD SUB- PRODUCTS}

\author{
Pablo Moreno ${ }^{1 *}$, Denis Rodrigue ${ }^{2}$, Yann Giroux ${ }^{2}$, Aldo Ballerini ${ }^{1}$, William Gacitúa ${ }^{1}$
}

\begin{abstract}
RESUMEN
El objetivo del presente estudio fue observar la morfología y caracterizar las propiedades mecánicas de materiales compuestos de madera y plásticos reciclados espumados. Se mezcló polietileno de alta densidad reciclado con fibra de madera de Pinus radiata D.Don (pino radiata) de 60 mesh. Las concentraciones de fibra fueron de 15 y $25 \%$ en peso, el polímero se espumó con un agente químico ACA (azodicarbonamida) y se utilizó anhídrido maleico MAPE (Epolene E-20) como agente de acoplamiento. Los compuestos se obtuvieron usando un proceso de extrusión en un equipo doble tornillo. Los resultados demostraron que el agente acoplamiento tuvo un efecto positivo sobre la adherencia del polímero reciclado y la fibra de madera. La densidad de los compuestos disminuyó con el agente espumante y las propiedades mecánicas de dureza, impacto, módulo de elasticidad en flexión y tracción fueron superiores para los compuestos no espumados. Se concluyó que al aumentar la concentración de fibra aumentaron las propiedades mecánicas de los compuestos a excepción de la tenacidad que al incorporar el agente espumante disminuyó ligeramente.
\end{abstract}

Palabras claves: Materiales compuestos madera-plástico, polietileno reciclado, azodicarbonamida, propiedades mecánicas, pino radiata.

\section{ABSTRACT}

The objective of the present study was to observe the morphology and characterize the mechanical properties of wood plastic composite foams. Recycled high density polyethylene was mixed with Pinus radiata D. Don (radiata pine) wood fiber of 60 mesh. The fiber concentrations were 15 and $25 \%$ by weight. The compounds were foamed with a chemical agent ACA (azodicabonamide) and MAPE (Epolene E-20) was used as a coupling agent. The composites were obtained by twinscrew extrusion. The results showed that the coupling agent had a positive effect on the adhesion of the recycled polymer and wood fiber. The density of the compounds decreased with blowing agent and the mechanical properties of hardness, impact, modulus of elasticity in bending and tension were higher for unfoamed composites. It was concluded that increasing the concentration of fiber increases the mechanical properties with the exception of impact resistance of the compounds that by incorporating the blowing agent decreased slightly.

Keywords: Wood plastic composite, recycled polyethylene, azodicarbonamide, mechanical properties, radiata pine. 


\section{INTRODUCCIÓN}

En la actualidad a nivel mundial existe una creciente búsqueda de nuevos materiales que puedan reemplazar a los tradicionales utilizados en la construcción como la madera, plástico y aluminio, entre otros, que sean de menor costo y presenten propiedades y características mejores o más eficientes. Los compuestos fabricados con aserrín de madera y polietileno se han destacado entre los productos considerados de post-consumo para la fabricación de compuestos madera-plástico.

Los materiales compuestos con fibra de madera y plástico presentan un aumento de la rigidez y bajos costos resultante del producto y son actualmente usados en la industria de la construcción para revestimiento, marcos para puertas y ventanas y cubiertas como reemplazo a la madera tradicional tratada a presión. Estos productos son resistentes a la humedad e insectos y no presentan pudrición, deformación, astillados, comparada con la madera tradicional (Li y Matuana 2003).

Mengeloglu y Matuana (2004), Faruk et al. (2007) y Klyosov (2007) indican que los compuestos madera-plástico presentan ciertos inconvenientes como el ser más frágiles y tener menos resistencia al impacto que los productos de plástico virgen. De igual manera, la densidad de los compuestos maderaplástico es superior a ciertos plásticos y es casi el doble a la madera solida. Debido a estas deficiencias, la utilización de estos compuestos es limitada y están impidiendo que sean competitivos y alcancen su pleno potencial en el mercado convencional de la madera estructural y en otras aplicaciones no estructurales.

Sustanciales esfuerzos hoy día se están realizando para mejorar las deficiencias que presentan los compuestos madera-plásticos. Recientemente, el concepto de la creación de estructuras celulares espumadas está siendo incorporada a través de procesos de extrusión e inyección y se ha demostrado mejoras significativamente en estas deficiencias (Faruk et al. 2007, Rivzi et al. 2008). Debido a la presencia de burbujas, la densidad del compuesto madera-plástico se ha reducido considerablemente (reducción de peso), disminuyendo el costo e importantes mejoras en la ductilidad y resistencia al impacto se han logrado, como lo indican Matuana et al. (1996, 1997, 1999); Kishbaugh et al. (2002); Bledzki y Faruk (2006).

Rivzi et al. (2005) indican que al incrementar el contenido de madera, la viscosidad del fundido aumenta significativamente, provocando una dispersión con mayor dificultad de la fibra de madera en la matriz polimérica. Esto afecta el proceso por un incremento en la presión y en la viscosidad en el cabezal y no permite una buena dispersión de la fibra de madera en la matriz para poder conseguir buenas muestras. Como se ha comprobado, la fibra de madera no puede ser espumada y si se incrementa la cantidad o proporción de madera disminuirá la fracción de vacíos total de las muestras, afectando el proceso de espumado tal como es reportado por Gosselin et al. 2006.

Matuana et al. (1999) y Guo et al. (2008) trabajaron con compuestos madera plástico virgen, incorporando espumantes y apuntaron que las propiedades mecánicas del material, como resistencia al impacto y a la tracción, mejoran con la adición del agente espumante. Bledzki y Faruk (2006) fabricaron compuestos microcelulares utilizando fibra de madera de conífera y latifoliadas al 30 y $50 \%$ de peso, polipropileno virgen como matriz y agentes espumantes exotérmicos, endotérmicos y endo/exotérmicos en una concentración del 4\%. Encontraron que la resistencia a la tracción se reduce proporcionalmente al incorporar los espumantes en comparación a los compuestos no espumados. Adicionando agente acoplante polipropileno maleatado (MAPP) al 5\%, con un 30\% de concentración de fibra de madera de latifoliadas, la resistencia a la tracción específica se incrementó un $80 \%$ con el agente espumante exotérmico. 
El uso de espumantes con agentes de acoplamiento podrían mejorar significativamente las propiedades mecánicas. El comportamiento mecánico de los compuestos microcelulares (espumados), dependen del tamaño fino y distribución de las células, lo cual muestra mejoras significativas en las propiedades mecánicas (Bledzki y Faruk 2006).

Doroudiani et al. (1998) fabricaron materiales compuestos microcelulares de polietileno de alta densidad (HDPE) y polipropileno isotáctico (PP) en condición virgen, usando $\mathrm{CO}_{2}$ como espumante y encontraron que debido a su morfología y cristalinidad, los polímeros mezclados produjeron una espuma más fina y uniforme que en cada polímero por separado, dando lugar a mejoras en las propiedades mecánicas, especialmente al impacto.

Lundin et al. (2004), Ashori y Nourbakhsh (2008), Adhikary et al. (2008), Ashori y Nourbakhsh (2009), todos indican que existe en la actualidad una cantidad de trabajos realizados con diversas poliolefinas en condición virgen, reforzados con fibra de madera y otras fibras de origen natural, utilizando sistemas de extrusión e inyección. El polietileno representa casi un $70 \%$ de los plásticos utilizados en la manufactura de los materiales compuestos madera plástico. El campo del reciclaje de polímeros ha sido objeto de varias investigaciones durante los últimos treinta años, pero sólo unos pocos trabajos están relacionados con el espumado de polímeros reciclados. El estado del arte indica un vacío existente de información respecto a la espumación de termoplásticos reciclados reforzados con sub-productos de la madera por los diferentes sistemas de producción y se podría asegurar que los pocos trabajos que podrían haberse desarrollados por extrusión no han sido publicados o aún no se han realizado.

El presente estudio permitirá examinar el potencial uso de sub-productos de la madera como lo es la fibra de pino radiata y el uso de una matriz reciclada compuesta por una mezcla de dos polímeros inmiscibles para la fabricación de materiales compuestos madera-plásticos espumados por extrusión, observar la morfología, caracterizar las propiedades mecánicas de los compuestos y aportar resultados al campo de la investigación científica en el área de compuestos madera plásticos reciclados espumados.

\section{MATERIALES Y MÉTODOS}

El polímero termoplástico reciclado utilizado en este estudio fue donado por el Laboratorio de Ingeniería Química de la Université Laval, Quebec, Canadá; en los mismos laboratorios se realizaron la fabricación y pruebas del compuesto. Se realizó un análisis de calorimetría diferencial de barrido (DSC) en base a tres réplicas, para conocer la composición y concentración del termoplástico reciclado a través de las transiciones térmicas en un intervalo de temperaturas entre los $20^{\circ} \mathrm{C}$ y $300^{\circ} \mathrm{C}$ a una velocidad de calentamiento de $10^{\circ} \mathrm{C} / \mathrm{min}$, se calculó la superficie de cada endoterma y la entalpía de fusión (mJ). La masa total de la mezcla se obtuvo sumando las entalpías resultantes de cada endoterma $(\mathrm{mJ})$ y se obtuvo el porcentaje en masa de cada polímero presente en la mezcla, el cual estaba conformado en promedio por un $82,3 \%$ de polietileno de alta densidad (HDPE) y un $17,7 \%$ de polipropileno $(\mathrm{PP})$ reciclados (Figura 1 ).

La mezcla de los polímeros presentó una densidad de $930 \mathrm{~kg} / \mathrm{m}^{3}$ y un índice de fluidez de $6,4 \mathrm{~g} / 10$ min, medido en base a ASTM D1238 condición $230^{\circ} \mathrm{C} / 2,16 \mathrm{~kg}$. El agente acoplante fue un MAPE, polietileno maleatado Epolene E-20 (peso molecular $7500 \mathrm{~g} / \mathrm{mol}$ ) de Westlake Chemical Corporation (USA). Similarmente, Celogen 754A (azodicarbonamida activada, ACA) en forma de polvo, obtenida de ChemPoint (USA), fue usado como agente espumante. La fibra de pino radiata fue proporcionada por el Centro de Biomateriales y Nanotecnología $(\mathrm{CBN})$ de la Universidad del Bío-Bío. El aserrín de madera fue tamizado y se utilizó el retenido en 60 mesh ( 250 micrones). La partícula retenida fue secada hasta alcanzar una humedad inferior al 2\%. Se utilizó un extrusor de doble tornillo co-rotativo (Leistritz 
ZSE-27) equipado con 10 zonas de calentamiento y un cabezal plano con dimensiones del perfil extruido de $152,4 \mathrm{~mm}$ de ancho y $6,35 \mathrm{~mm}$ de altura.

En un primer paso el polímero reciclado fue extruido con y sin agente espumante, posteriormente se extruyeron los compuestos madera-plástico variando la concentración de la fibra en $15 \%$ y $25 \%$, manteniendo constante la concentración de agente acoplante, base a peso de madera seca, en 5\%, y $0.2 \%$ para el agente espumante en base al peso del polímero. Se fabricaron un total de 10 formulaciones como se indica en la tabla 1.

Tabla 1. Formulaciones de polímero reciclado y compuestos madera plástico con y sin aditivos.

\begin{tabular}{|c|l|}
\hline Código & \multicolumn{1}{|c|}{ Formulación del compuesto } \\
\hline F1 & Polímero reciclado \\
\hline F2 & Polímero reciclado $+15 \%$ fibra de madera \\
\hline F3 & Polímero reciclado $+25 \%$ fibra de madera \\
\hline F4 & Polímero reciclado $+15 \%$ fibra de madera $+5 \%$ MAPE \\
\hline F5 & Polímero reciclado $+25 \%$ fibra de madera $+5 \%$ MAPE \\
\hline F6 & Polímero reciclado $+0.2 \%$ ACA \\
\hline F7 & Polímero reciclado $+15 \%$ fibra de madera $+5 \%$ MAPE $+0.2 \%$ ACA \\
\hline F8 & Polímero reciclado $+25 \%$ fibra de madera $+5 \%$ MAPE $+0.2 \%$ ACA \\
\hline F9 & Polímero reciclado $+15 \%$ fibra de madera $+0.2 \%$ ACA \\
\hline F10 & Polímero reciclado $+25 \%$ fibra de madera $+0.2 \%$ ACA \\
\hline
\end{tabular}

Las formulaciones del polímero sin reforzante (madera), con y sin espumante, fueron mezclados en un recipiente (mezclas en seco) e introducidas a la extrusora a través de un alimentador calibrado. Para el resto de las formulaciones, el polímero reciclado mezclado con las concentraciones fijas de aditivos, fue introducido por un alimentador calibrado y las concentraciones de fibra de madera en un $15 \%$ y $25 \%$, se introdujeron a la extrusora por otro alimentador calibrado. En todos los casos la velocidad de los tornillos de la extrusora se mantuvo en $100 \mathrm{rpm}$ y las temperaturas de la extrusora se mantuvieron en $165,170,170,175,180,185,190,185,180,175^{\circ} \mathrm{C}$ y $165^{\circ} \mathrm{C}$ en el cabezal. Las muestras extruidas que presentaron a la salida del cabezal un ancho de $120 \mathrm{~mm}$ y $3 \mathrm{~mm}$ de espesor, fueron enfriadas a temperatura ambiente, para definir la morfología de las formulaciones espumadas.

Para la determinación de la densidad se utilizó un picnómetro modelo Ultrapyc 1200e, para la resistencia a la tracción y el módulo de elasticidad (ASTM D 638) y para resistencia a la flexión y el módulo de elasticidad (ASTM D790) se utilizó una máquina universal Instron model 5565. Para la determinación de resistencia a la dureza se utilizó un durómetro análogo, modelo 307L tipo D (ASTM D2240), cuyas unidades de medición son expresadas en shore D y para la resistencia al impacto IZOD se uso un equipo Tinius Olsen Model 104. Las dimensiones de las muestras y velocidades de ensayo para cada propiedad fueron seleccionadas de acuerdo a las respectivas normas ASTM. Se utilizaron 5 probetas para la determinación de la densidad. Para la resistencia a la dureza se utilizó una probeta de $10 \mathrm{~cm}$ x $10 \mathrm{~cm}$ y se realizaron 10 lecturas en ambas caras de la muestra. Para la resistencia a la flexión se utilizaron 6 probetas. La resistencia a la tracción fue medida utilizando 6 probetas en el sentido longitudinal y para la resistencia al impacto, fueron utilizadas 15 probetas sin entalladura.

Para el análisis de fractura de los compuestos fabricados se cortaron pequeñas piezas rectangulares de la parte central de los compuestos, las cuales se sumergieron durante $30 \mathrm{~s}$ en nitrógeno líquido para 
evitar la deformación de la morfología y lograr una fractura frágil en el sentido longitudinal y transversal. Las muestras fueron recubiertas con una aleación de oro y platino, para luego ser inspeccionadas con un microscopio electrónico (SEM) marca JEOL modelo JSM840A. Se observó la superficie de los compuestos espumados fracturados, utilizando aumentos de 20X y 50X. Se utilizó un análisis de varianza de una vía (ANOVA) para examinar las diferencias en las medias de las variables respuestas de los compuestos madera plástico a las diferentes concentraciones de fibras con las diferentes formulaciones. Todos los análisis se realizaron utilizando un software estadístico Design Expert versión 8.0.

\section{RESULTADOS Y DISCUSIÓN}

En la figura 1, se muestra el análisis de calorimetría diferencial de barrido (DSC) el cual representa el comportamiento promedio del termoplástico reciclado. Mediante el análisis de la curva obtenida con el DSC, es dable comprobar que existen dos polímeros en la mezcla de plásticos reciclados, en este caso PE y PP (no hay otra contaminación de termoplásticos). Los dos picos claramente marcados en el perfil DSC están asociados con la presencia de estos dos polímeros; con la energía (área sobre la curva en los dos picos), se determinó el porcentaje en la mezcla de cada uno de ellos. En base a las temperaturas de fusión y a las endotermas, el polímero reciclado estaba conformado en promedio por una mezcla de dos polímeros termoplásticos, HDPE con un 82,3\% y PP con 17,7\%.

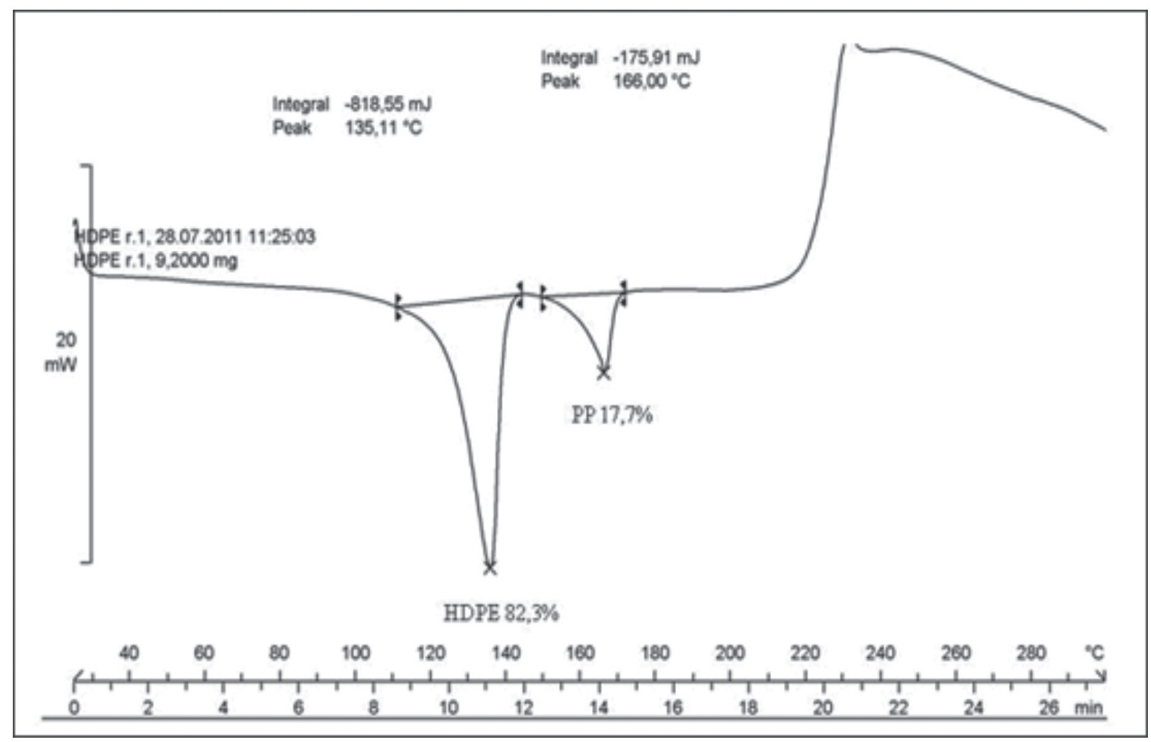

Figura 1. Análisis de Calorimetría Diferencial de Barrido (DSC) de la mezcla de polímeros reciclados.

En las figuras 2 a 6, se muestran las micrografias mediante SEM para el polímero con y sin espumante y materiales compuestos con y sin aditivos. En la figura 2, se puede observar en el polímero reciclado espumado la diversidad de tamaños de las burbujas, de formas elípticas, abiertas y cerradas, predominando las burbujas de gran tamaño en dirección al sentido del flujo del perfil extruido, que en el sentido transversal y esto se vío reflejado en la abrupta disminución de la densidad del polímero reciclado al ser espumado. Rodrigue y Gosselin (2002) encontraron un patrón similar en la preparación y caracterización de plásticos virgen espumados en moldeo por inyección, señalando que este comportamiento se debe a las condiciones de extrusión e inyección. Cuando las espumas son preparadas por extrusión o inyección, se considera una probable deformación en la dirección del flujo. 
Twite y Rodrigue (2007) estudiaron el efecto del reciclado sobre las propiedades del polietileno de baja densidad espumado, indicando que la reducción al cizalle y la viscosidad de elongación, como resultado de la degradación del polímero, sean responsable por el mayor número de células debido a una menor resistencia hidrodinámica en la etapa de crecimiento de las células, así como tambien mayor probabilidad de coalescencia de células.
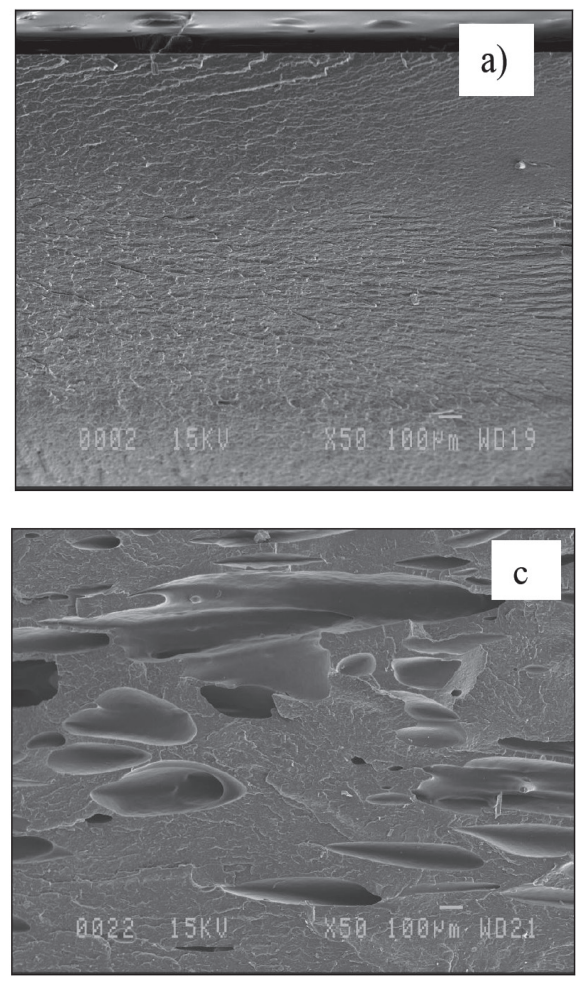
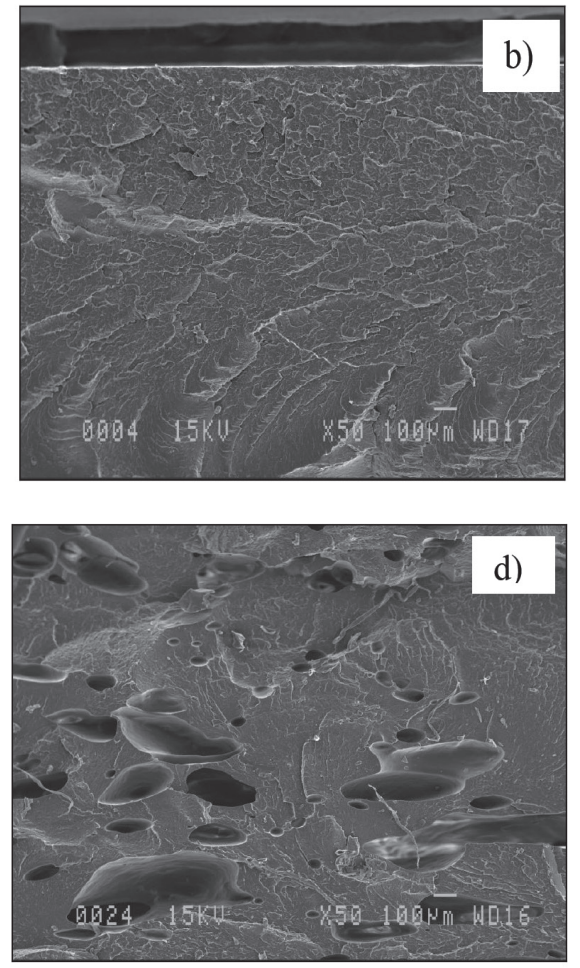

Figura 2. Análisis de fractura del polímero reciclado sin espumar (a-b) y polímeros reciclados espumados (c-d) en sentido longitudinal y transversal con 50X.

La figura 3, muestra un compuesto con $15 \%$ de fibra sin agente acoplante y sin agente espumante. Se pueden observar espacios vacíos generados por las fibras extraídas durante la tracción, así como una fractura de fibra perpendicular, siendo atribuible a la débil interacción interfacial entre las fibras de madera y la matriz. Por el contrario, cuando se utiliza MAPE (Figura 4), las muestras presentan una mejor interacción fibra-matriz. Al impregnarse las fibras con el pólimero por la presencia del agente acoplante, se reduce la cantidad de fibras extraídas o fracturadas (efecto "pull-out"). La figura 5 , muestra un compuesto al 15\% de fibra con MAPE y ACA. Se puede observar la buena integración generada por el agente de acoplamiento entre la fibra y la matriz y de igual manera fibras que se deformaron antes de fracturarse, quedando adheridas a la matriz que presenta burbujas en todo su volumen. Por el contrario, en la figura 6 , se presenta un compuesto espumado sin la incorporacion del MAPE. Se logra observar la falta de adhesión fibra-matriz, permitiendo que las fibras se deformen y rompan con facilidad.

Se puede observar que la fracción de espacios vacíos en el polímero reciclado fue significativamente más alta en comparación a la de los compuestos. El tamaño de las burbujas desarrolladas en los compuestos fue más pequeño que las desarrolladas en el polímero reciclado espumado. Estos resultados son esperables debido a la mayor rigidez y baja solubilidad del gas en los compuestos en comparación al polímero, que está de acuerdo con lo publicado en Li y Matuana (2003), investigadores que trabajaron en extrusión de compuestos madera plástico con polietileno de alta densidad en estado virgen. 

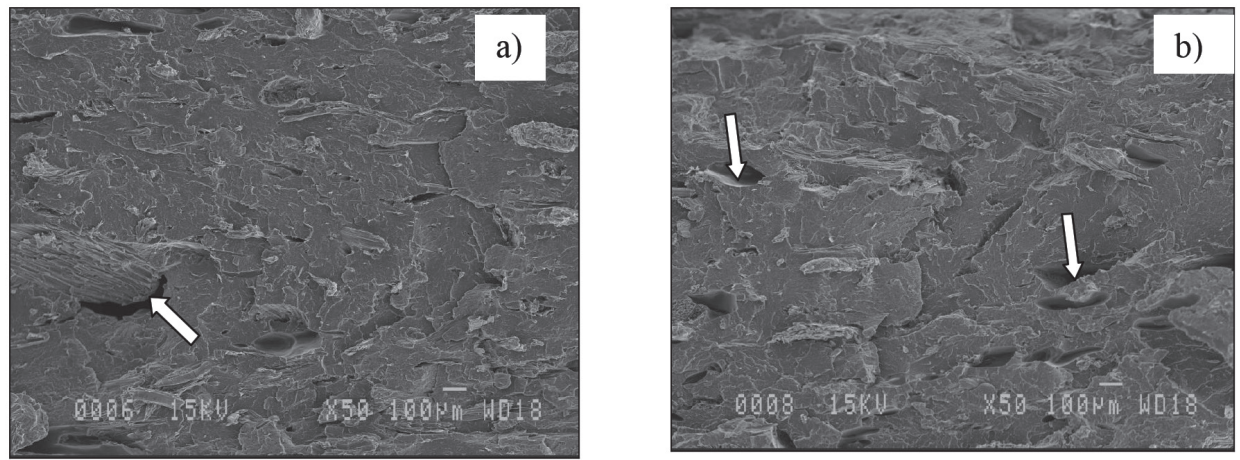

Figura 3. Análisis de fractura del compuesto madera plástico reciclado espumado con $15 \%$ de fibra, sin MAPE $\sin$ ACA, 50X.

a) sentido longitudinal y b) sentido transversal.
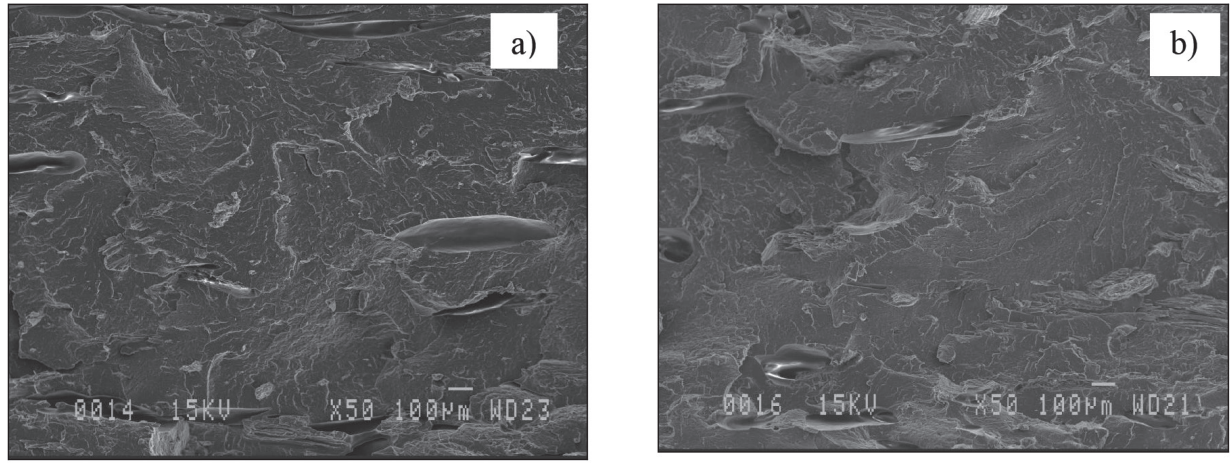

Figura 4. Análisis de fractura del compuesto madera plástico reciclado espumado con $15 \%$ de fibra, $5 \%$ MAPE, $50 X$.

a) sentido longitudinal y b) sentido transversal.
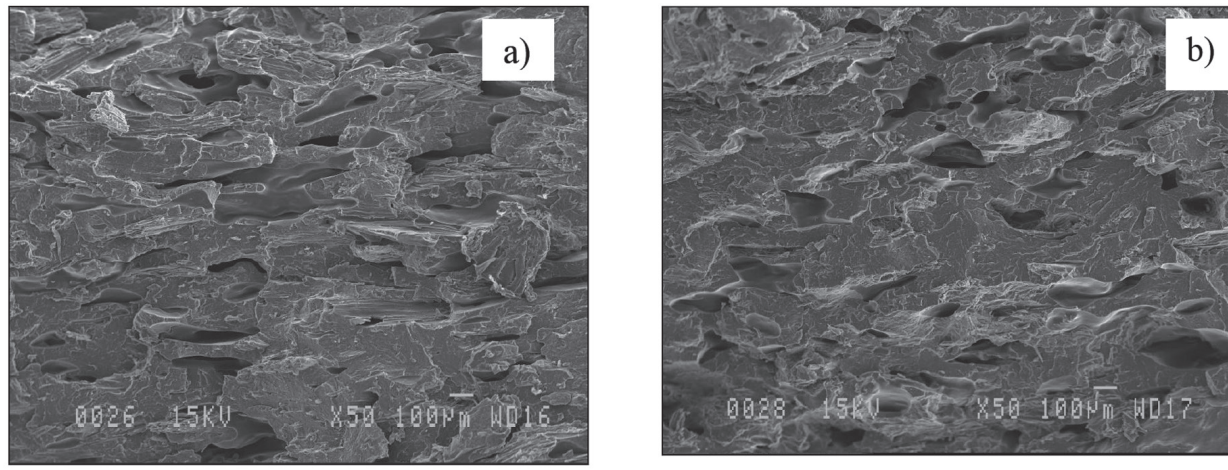

Figura 5. Análisis de fractura del compuesto madera plástico reciclado espumado con $15 \%$ de fibra, $5 \%$ MAPE, $0.2 \%$ ACA, 50X.

a) sentido longitudinal $\mathrm{y} b$ ) sentido transversal. 

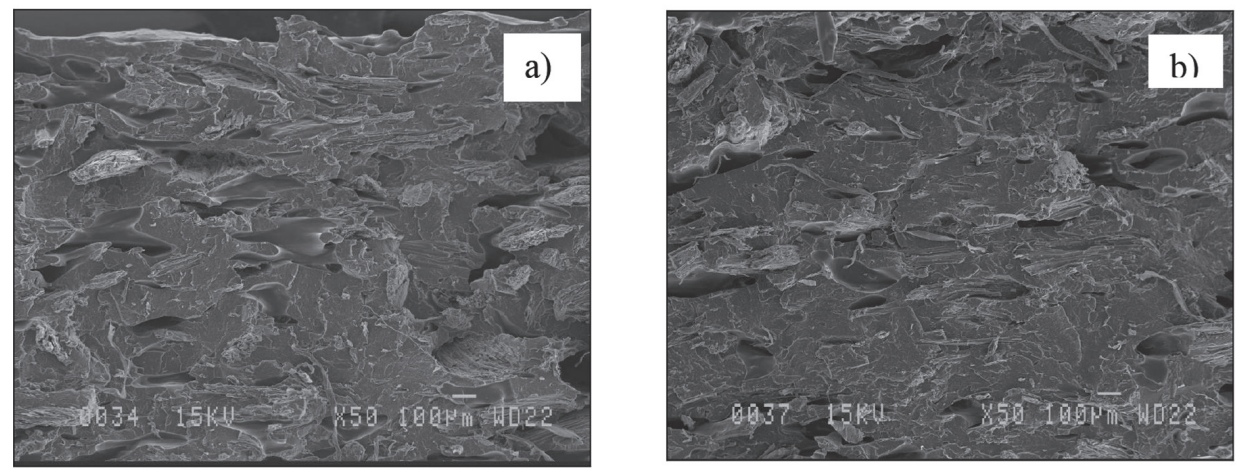

Figura 6. Análisis de fractura del compuesto madera plástico reciclado espumado con $15 \%$ de fibra, $0.2 \%$ ACA, $50 \mathrm{X}$.

a) sentido longitudinal $\mathrm{y}$ b) sentido transversal.

La figura 7, muestra el efecto de la cantidad de fibras de madera, la incorporación del agente de acoplamiento y el agente espumante sobre la densidad del polímero reciclado y los compuestos madera-plástico. Los resultados del análisis de varianza muestran que el valor de probabilidad "p" fue menor que 0.05 para el modelo, lo que indica que el modelo es significativo.

Se observa que el polímero reciclado (F1) obtuvo una densidad de $938 \mathrm{~kg} / \mathrm{m}^{3}$; por otro lado los compuestos madera-plástico sin la incorporación de aditivos (F2 y F3) con concentración de fibras de $15 \%$ tuvieron una densidad de $979 \mathrm{~kg} / \mathrm{m}^{3}$; al aumentar la cantidad de fibra a $25 \%$ la densidad aumentó a $990 \mathrm{~kg} / \mathrm{m}^{3}$. Al añadir el agente de acoplamiento MAPE en las formulaciones F4 y F5, la densidad para $15 \%$ de fibra fue de $998 \mathrm{~kg} / \mathrm{m}^{3}$ y de $1005 \mathrm{~kg} / \mathrm{m}^{3}$ para $25 \%$ de fibra. Se puede observar que al aumentar la concentración de fibra y al incorporar el agente de acoplamiento la densidad tiende a aumentar. Esta situación puede darse debido a que al aumentar la cantidad de fibra existe mayor densificación del compuesto al colapsar plásticamente las fibras de madera por las altas presiones y temperaturas al interior del barril de la extrusora, de igual manera el efecto que ejerce el agente acoplante entre la matriz y el relleno, permite una mejor adhesión interfacial, mejor contacto, evitando que se puedan desarrollar espacios vacíos y microfracturas internas en el compuesto que puedan acumular burbujas de aire y vapor de agua que afecten la densidad del producto. Para el polímero reciclado con agente espumante (F6) se observa una disminución de la densidad de $938 \mathrm{~kg} / \mathrm{m}^{3}$ a $801 \mathrm{~kg} / \mathrm{m}^{3}$. Por otro lado, en los compuestos con MAPE y ACA (F7 y F8) la densidad fue de $962 \mathrm{~kg} / \mathrm{m}^{3}$ y de $982 \mathrm{~kg} / \mathrm{m}^{3}$ para $15 \%$ y $25 \%$ de fibra, respectivamente. Dado que la fibra de madera no puede ser espumada, un incremento en la cantidad de madera en el compuesto necesariamente disminuye la fracción de espacios vacios total. Se observó el efecto positivo del agente espumante al disminuir la densidad de los compuestos. Para los compuestos F9 y F10, sólo se incorporó el ACA y la densidad disminuyó respecto a los compuestos no espumados (F2 y F3), resultando densidades del compuesto de $872 \mathrm{~kg} / \mathrm{m}^{3}$ y $922 \mathrm{~kg} / \mathrm{m}^{3}$ para $15 \%$ y $25 \%$ de fibra, respectivamente. Estos resultados son similares a los encontrados por Zhang et al. (2005) quienes manifiestan que al aumentar la concentración de la fibra aumenta la densidad de los compuestos. El incremento de la fracción de espacios vacíos de los compuestos espumados con el agente acoplante puede ser atribuido a la mejora de la adhesión interfacial entre el polímero y la fibra de madera. Cuando la adhesión entre la matriz y el reforzante es mejorada a través del uso de agentes acoplantes, más gas es disuelto en las muestras y la estructura espumada es mejor desarrollada, evitando que el gas se escape por la microfracturas de la interface, por consiguiente existe una disminución en la densidad. Estos resultados están de acuerdo con los encontrados en la literatura. Sin embargo, la inefectividad del agente de acoplamiento con ciertos agentes espumantes todavía no ha sido bien explicada (Li y Matuana 2003).

Además, el análisis de varianza deja de manifiesto que existe una interacción entre los factores de agente acoplante y agente espumante, produciendo cambios en la densidad media del material. Zuñiga 
(2010) encontró valores similares de densidad en un proceso de extrusión de perfiles espumados de madera plástico, utilizando como matriz polipropileno virgen, fibra de madera de pino radiata, MAPP como agente acoplante e Hydrocerol como agente espumante. Soberanes et al. (2005) mencionan que no se tiene un control en la densidad en el proceso de extrusión, provocando mayor cantidad de espacios y obteniendo con esto que pueda escaparse el gas producido por el agente espumante y no tener una morfología homogénea. Gosselin et al. (2006) trabajaron en la morfología de compuestos madera plástico reciclado por moldeo en inyección y mencionan que al aumentar la concentración de fibra se puede estar logrando aumentar la viscosidad de la mezcla fundida, provocando una reducción en el tamaño promedio de células.

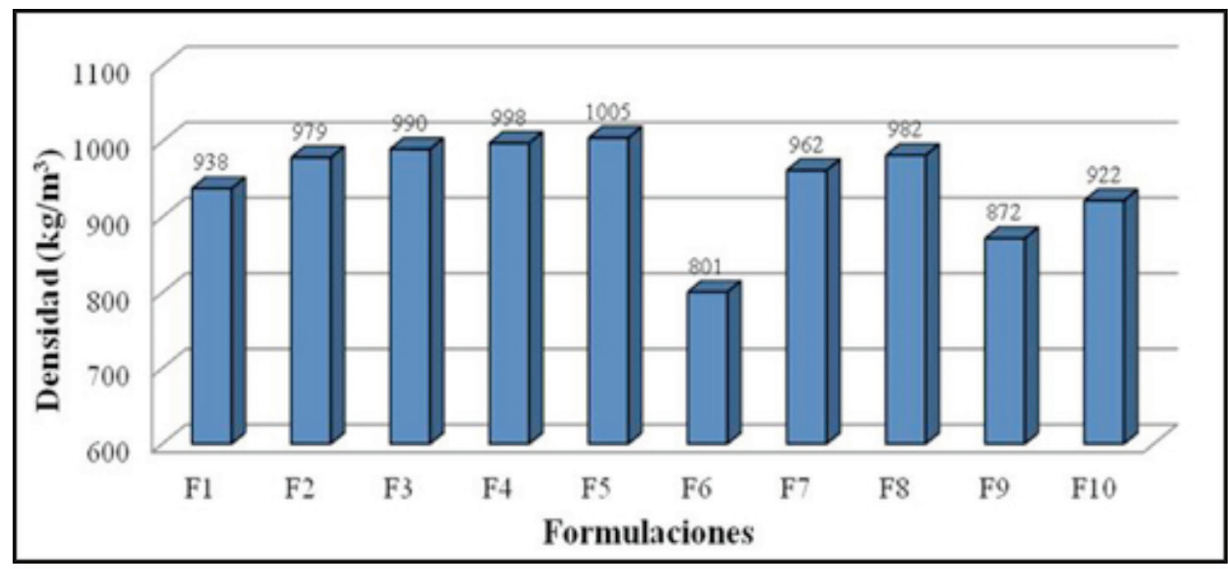

Figura 7. Densidad como función de la concentración de fibra en polímeros reciclados, reforzados con fibra de pino radiata, con y sin aditivos.

La figura 8, muestra el efecto de la concentración de la fibra de madera, la incorporación del agente de acoplamiento y el agente espumante sobre la dureza del polímero reciclado y los compuestos maderaplásticos. Los resultados del análisis de varianza muestran que el valor de probabilidad "p" fue mayor que 0.05 para el modelo, lo que indica que no existen diferencias significativas. Se puede apreciar tanto para el polímero sin agente espumante y los compuestos madera plástico con y sin agente acoplante, que los valores de dureza fueron superiores a aquellos en los cuales se incorporó el agente espumante. La dureza intrínsecamente es una condición de la superficie del material, la cual es fuertemente dependiente del porcentaje de madera presente en el compuesto. Al comparar los compuestos F5 y F8, no se observa un efecto significativo del agente espumante (agregado en un $0.2 \%$ en el compuesto F8) sobre la densidad del compuesto. 


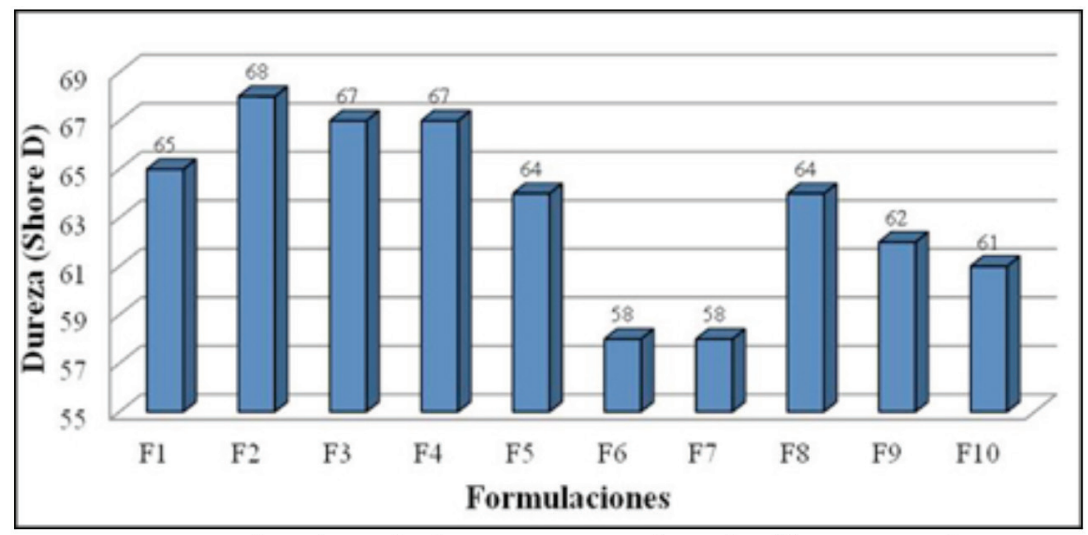

Figura 8. Dureza como función de la concentración de fibra en polímeros reciclados, reforzados con fibra de pino radiata, con y sin aditivos.

La figura 9, muestra el efecto de la concentración de la fibra de madera, la incorporación del agente de acoplamiento y el agente espumante sobre la resistencia al impacto del polímero reciclado y los compuestos madera-plásticos. Los resultados del análisis de varianza muestran que el valor "p" fue menor que 0.05 para el modelo, lo que indica que este es significativo. Se aprecia que la mayor resistencia al impacto la presenta el polímero reciclado, siendo una de las grandes cualidades del polietileno de alta densidad en comparación a los compuestos madera-plástico. Se puede apreciar que el aumento en la concentración de fibra de madera de $15 \%$ a $25 \%$ provoca que la energía a la fractura del compuesto disminuya, presentando menor resistencia al impacto los compuestos con $25 \%$ de concentración de fibra de madera con y sin MAPE. Estos resultados son similares a los encontrados por Raygoza y Gastinel (2009) quienes estudiaron el efecto de la fibra de agave de desecho en el refuerzo de polipropileno virgen y reciclado. En relación a la presente investigación, en el polímero reciclado, la disminución de la resistencia fue bastante notoria y alcanzó un 19\% respecto al polímero no espumado. Se puede observar que la presencia del agente espumante en los compuestos tendría un efecto negativo, disminuyendo la resistencia al impacto respecto a los no espumados. La concentración de la fibra y del agente espumante por separados afecta en forma significativa la resistencia promedio al impacto. Estos resultados pudieron manifestarse por la presencia de burbujas grandes en la matriz polimérica, reduciendo el área de la sección real de las muestras, los espacios vacíos y el tipo de celda formada y no ofrecieron resistencia al momento del impacto, por donde la energía pudo disiparse, facilitando la deformación y fractura más rápida de la muestra. 


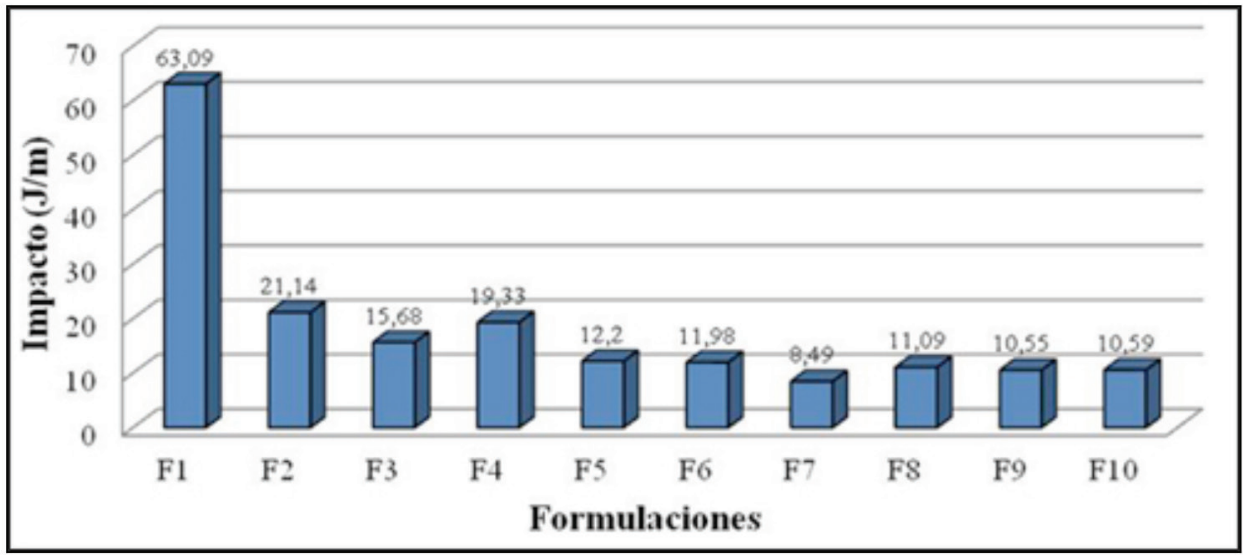

Figura 9. Resistencia al impacto como función de la concentración de fibra en polímeros reciclados, reforzados con fibra de pino radiata, con y sin aditivos.

La figura 10, muestra el efecto de la cantidad de fibra de madera, la incorporación del agente de acoplamiento y el agente espumante sobre el módulo de elasticidad en flexión y tracción del polímero reciclado y los compuestos madera-plásticos. Los resultados del análisis de varianza muestran que el valor " $p$ " fue menor que 0.05 , lo que permite establecer que existen diferencias significativas. La concentración de la fibra, el agente acoplante y el agente espumante por separados afectan de forma significativa la resistencia promedio a la flexión y tracción. Se puede observar un aumento de los módulos de flexión y tracción de los compuestos respecto al polímero reciclado. El módulo de elasticidad en flexión se incrementa al aumentar la cantidad de fibra de $15 \%$ a $25 \%$ (946.8 MPa a 1012.8 MPa), mejorando la resistencia final de los compuestos al incorporar agente acoplante (1071.9 $\mathrm{MPa}$ a $1113.4 \mathrm{MPa})$. En relación al módulo de elasticidad en tracción se observa el mismo comportamiento que en flexión, el módulo en tracción aumenta al incorporar fibra de madera de $15 \%$ a $25 \%(374.7 \mathrm{MPa}$ a $386.6 \mathrm{MPa})$ y al incorporar agente acoplante existió un aumento evidentemente significativo (380.5 MPa y 399.4 MPa). El patrón de desempeño en tracción de los compuestos con y sin aditivos puede verse en la figura 11. En ella se puede observar las curvas esfuerzo-deformación promedio de las 10 formulaciones evaluadas; se destaca la gran deformación hasta la ruptura que muestra el polímero reciclado sin aditivos (F1). El compuesto más frágil resultó ser el fabricado según la formulación F8, con bajos valores de esfuerzo y deformación en ruptura.

Se puede apreciar que el polímero reciclado (F1) presentó el mayor porcentaje de deformación promedio a la ruptura (superior al $20 \%$ ), con un esfuerzo máximo a la ruptura de $22.6 \mathrm{MPa}$, respecto al polímero reciclado espumado (F6), quien mostró menor deformación a la ruptura de 15,6\%, con un esfuerzo máximo a la ruptura de 16.0 MPa. Los compuestos madera-plástico sin aditivos (F2 y F3) con $15 \%$ de fibra presentaron mayor deformación y esfuerzo al quiebre que los compuestos con $25 \%$ de fibra. Caso similar se presentó en los compuestos sin espumar con incorporación del agente acoplante. Se puede observar una fuerte caída en los valores del esfuerzo máximo a la ruptura en los compuestos con agente espumante y agente acoplante (F7 y F8) y sin agente acoplante (F9 y F10), se observa de igual manera que los compuestos con el porcentaje de $15 \%$ presenta mayor deformación a la ruptura que con $25 \%$. Estos resultados son similares a los obtenidos por Adhikary et al. (2008) quienes trabajaron con polietileno de alta densidad virgen y reciclados sin espumación. Las mejores propiedades mecánicas de los compuestos con agente acoplante se atribuye principalmente a la formación de enlaces interfaciales entre la fibra y la matriz, permitiendo llevar a cabo un esfuerzo reforzante, aunque se provoca una rigidización al ir aumentando la cantidad de fibra en el compuesto (Qui et al. 2004, Mohanty et al. 2004, Lu et al. 2005, Raygoza y Gastinel 2009). Se puede observar que la presencia del agente espumante, 
podría tener un efecto negativo, disminuyendo la resistencia a la tracción y flexión del polímero reciclado y de los compuestos. Resultados similares fueron encontrados por Soberanes et al. (2005) en compuestos con polietileno de baja densidad virgen. Para este estudio, las disminuciones encontradas no fueron significativas en relación a los compuestos no espumados, esto puede ser atribuido a la distribución de las fibras y burbujas dentro de las muestras, de igual manera se debe considerar que la matriz polimérica es un polímero de post-consumo y que tal como fue confirmado con análisis con DSC, el polímero reciclado es una mezcla de tipos de polímeros y otras, que podrían estar ocasionando modificaciones en su estructura en comparación de un polímero en condición virgen.

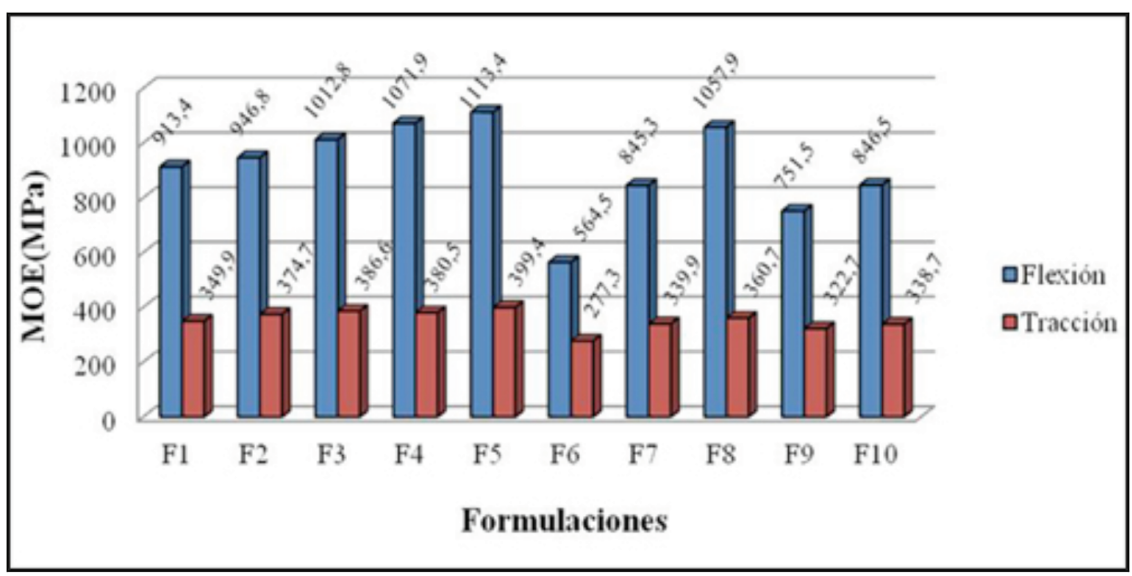

Figura 10. Módulo de flexión y tracción, como función de la concentración de fibra en polímeros reciclados, reforzados con fibra de pino radiata, con y sin aditivos.

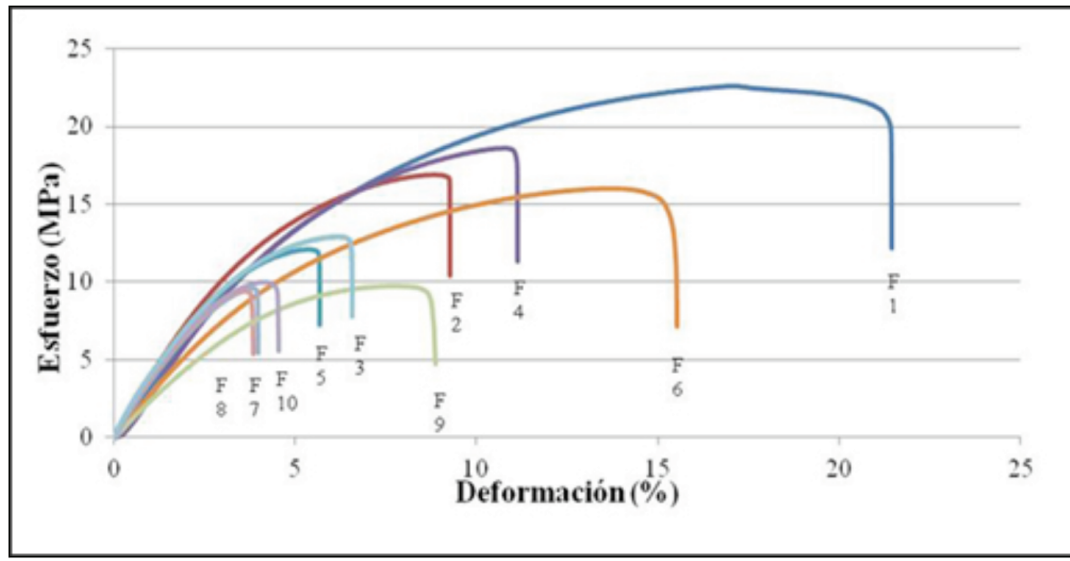

Figura 11. Curva de esfuerzo versus deformación en tracción, como función de la concentración de fibra en polímeros reciclados, reforzados con fibra de pino radiata, con y sin aditivos. 


\section{CONCLUSIONES}

En este trabajo, se estudiaron y discutieron las propiedades mecánicas de materiales compuestos de madera y plásticos reciclados espumados por extrusión en relación con su morfología y densidad. Se observó que el uso de un agente de acoplamiento es escencial para mejorar significativamente el enlace interfacial entre la matriz y el reforzante.

La incorporación de un agente espumante redujo la densidad del polímero reciclado de $938 \mathrm{~kg} / \mathrm{m}^{3}$ a $801 \mathrm{~kg} / \mathrm{m}^{3}$. En los compuestos con 15\% y $25 \%$ de fibra con agente espumante sin la incorporación del agente de acoplamiento, la densidad fue de $872 \mathrm{~kg} / \mathrm{m}^{3}$ y $922 \mathrm{~kg} / \mathrm{m}^{3}$ respectivamente.

Se encontró que los módulos de elasticidad en tracción y flexión, presentaron un aumento al aumentar la concentración de fibra a $25 \%$ y al incorporar el agente acoplante, disminuyendo ligeramente al incorporar agente espumante.

Los valores de la energía media del impacto disminuyen al aumentar la concentración de la fibra de madera de $15 \%$ a $25 \%$, y de igual manera al agregar el agente espumante. La dureza se ve disminuida al incorporar el agente espumante en comparación con los compuestos sin aditivos.

Se pudo constatar que las variables del proceso y las características de las materias primas juegan un papel importante en la generación de estructuras microcelulares complejas en los compuestos, y en función de ello, el logro de un producto que reúna buenas propiedades físicas y mecánicas. Sin embargo, la inefectividad del agente de acoplamiento con ciertos agentes espumantes no se puede explicar bien hasta el momento, situación que abre nuevas rutas de investigación.

\section{AGRADECIMIENTOS}

Los autores agradecen al personal del Laboratorio de Ingeniería Química de la Université Laval, Quebec, Canadá, al Centro de Biomateriales y Nanotecnología (CBN) y al Centro de Polímeros Avanzados (CIPA) de la Universidad del Bío-Bío, por el apoyo brindado en el desarrollo de esta investigación.

\section{BIBLIOGRAFÍA}

Adhikary, K.; Pang, S.; Staiger, M. 2008. Dimensional Stability and Mechanical Behavior of wood-plastic composites based on recycle and virgin high-density polyethylene (HDPE). Composites Part B: Engineering 39(5):807-815.

Ashori, A.; Nourbakhsh, A. 2008. A comparative study on mechanical properties and water absorption behavior of fiber-reinforced polypropylene composites prepared by OCC fiber and aspen fiber. Polymer Composites 29(5):574-578.

Ashori, A.; Nourbakhsh,A. 2009. Characteristics of wood-fiber plastic composites made of recycled materials. Waste Management 29(4):1291-1295.

Bledzki, A.; Faruk, 0. 2006. Microcellular injection molded wood fiber-PP composites: Part I-effect of chemical foaming agent content on cell morphology and physic-mechanical properties. Journal of Cellular Plastic 42(1):63-76.

Doroudiani, S.; Park, C.; Kortschot, M. 1998. Processing and Characterization of Microcellular foamed High-density Polyethylene/Isotactic Polypropylene Blends. Polymer Engineering Science 38(7):1205-1215.

Faruk, O.; Bledzki, A.; Matuana, L. 2007. Microcellular foamed wood-plastic composites by different processes: A review. Macromolecular Materials and Engineering 292(2):113-127.

Gosselin, R.; Rodrigue, D.; Riedl, B. 2006. Injection Molding of postconsumer wood-plastic composites I: Morphology. Journal of Thermoplastic Composite Materials 19(6):639-657. 
Guo, G.; Lee, Y.; Rivzi, G.; Park, C. 2008. Influence of wood fiber size on extrusion foaming of wood fiber/ HDPE composites. Journal of Applied Polymer Science 107(6):3505-3511.

Kishbaugh, L.; Xu, J.; Casale, M. 2002. Prediction of the mechanical properties with part weight reduction for injection molding microcellular foam parts, SPE ANTEC tech. paper 2:1905-1909.

Klyosov, A. 2007. Wood-Plastic Composite. Wiley-Interscience Inc., Hoboken, New Jersey. USA.

Li, Q.; Matuana, L. 2003. Foam extrusion of high density polyethylene/Wood-flour composites using chemical foaming agents. Journal of Applied Polymer Science 88(14):3139-3150.

Lu, J.; Negulescu, I.; Wu, Q. 2005. Maleated Wood fiber/high density polyethylene composites: Coupling mechanism and interfacial characterization. Composites Interfaces 12(1-2):125-140.

Lundin, Y.; Cramer, S.M.; Falk, R.H.; Felton, C. 2004. Accelerated weathering of natural fiber-filled polyethylene composites. Materials in Civil Engineering 16(4):351-363.

Matuana, L.; Park, C.; Balatinecz, J. 1996. Characterization of microcellular foamed PVC/cellulosic-fibre. Journal of Cellular Plastics 32(5):449-469.

Matuana, L.; Park, C.; Balatinecz, J. 1997. Processing and cell morphology relationships for microcellular foamed PVC/wood fibre composites. Polymer Engineering \& Science 37(7):1137-1147.

Matuana, L.; Balatinecz, J.; Park, C. 1999. Foaming of woodfiber-plastic composites. The Fifth International Conference on Woodfiber-Plastic Composites. Madison, Wisconsin. USA.

Mengeloglu, F.; Matuana, L. 2004. Mechanical properties of extrusion-foamed rigid PVC/wood-flour composites. Journal of Vinyl and Additive Technology 9(1):26-31.

Mohanty, S.; Verna, S.; Nayak, S.; Tripathy, S. 2004. Influence of fiber treatment on the performance of sisal-polypropylene composites. Journal of Applied Polymer Science 94(3):1336-1345.

Qui, W.; Endo, T.; Hirotsu, T. 2004. Interfacial interaction of a novel mechanochemical composite of cellulose with maleated polypropylene. Journal of Applied Polymer Science 94(3):1326-1335.

Raygoza, R.; Gastinel, J. 2009. Efecto de la fibra de agave de desecho en el reforzamiento de polipropileno virgen o reciclado. Revista Mexicana de Ingeniería Química 8(3):319-327.

Rivzi, G.; Guo, G.; Park, C.; Kim, Y. 2005. Critical issues in extrusion foaming of plastic/wood fiber composites (Review). Cellular Polymers 24(6):347-362.

Rivzi, G.; Park, C.; Guo, G. 2008. Strategies for processing wood plastic composites with chemical blowing agents. Journal of Cellular Plastics 44(2):125-137.

Rodrigue, D.; Gosselin, R. 2002. The effect of calcium carbonate particle size on LDPE foam morphology. Proceedding of the 4th International Blowing Agents and Foaming Processes Conference.Heidelberg, Alemania, pp. $157-166$

Soberanes, D.; Quirarte, J.; Rodrigue, D.; Gonzalez, R. 2005. Polímeros compuestos espumados basados en fibra de agave. Memorias del XVIII Congreso Nacional de la Sociedad Polimérica de México. Puerto Vallarta, Jalisco, pp.443-448.

Twite, E.; Rodrigue, D. 2007. The effect of recycling on LDPE foam properties. Progress in Rubber. Plastics and Recycling Technology 24(1):1-17.

Zhang, S.; Rodrigue, D.; Rield, B. 2005. Preparation and morphology of polypropylene/Wood flour composite foam via extrusion. Polymer Composites 26(6):731-738.

Zúñiga, A. 2010. Optimización de un proceso de extrusión de perfiles espumados de madera platico mediante un análisis de superficie de respuesta. Tesis Magister. Universidad de Concepción, Concepción, Chile, 85 pp. 